\title{
TEÑIDO DE ALGODÓN CON PIGMENTO NATURAL DE YUCUSHAPANA UTILIZANDO DIFERENTES MORDIENTES ${ }^{36}$
}

Abraham Arsenio Palacios Velásquez

\author{
Facultad de Ingeniería Química de la Universidad Nacional del Centro del Perú
}

\begin{abstract}
RESUMEN
El presente proyecto constituye parte de la línea de investigación de proceso de transformación de recursos naturales que se ejecuta en la Facultad de Ingeniería Química de la UNCP. El objetivo de evaluar el potencial de tinción del tinte obtenido de la especie forestal yacushapana (terminalia oblonga) en la aplicación de tinción de algodón. La selva peruana cuenta con una gran riqueza vegetal, entre ellas, plantas que tienen sustancias activas del tipo colorante como flavonoides, xantonas, quinonas, carotenoides, etc., sustancias que pueden usarse en la industria cosmética, alimenticia y en la industria textil. El presente proyecto investigó la especie forestal con potencial para su uso en la industria textil en el teñido de fibras. Actualmente la comunidades nativas de la Selva baja de la Región Ucayali, utiliza extractos tintóreos acuosos de diversidad de plantas para el teñido de las fibras que se usan en la confección de diversidad de prendas de vestir y ropa de cama. Los mencionados extractos tintóreos acuosos son obtenidos de una manera empírica por lo que tienen una vida media muy baja y poca disponibilidad en algunas épocas del año; por lo que con los resultados del presente proyecto se podrá asesorar a pequeños empresarios de la industria textil en la extracción, secado, preservación y aplicación de tintes naturales. Antiguamente se usaban extractos tintóreos de plantas para teñir fibras, ropa, dar color a alimentos etc., lo que dejó de realizarse al aparecer los colorantes artificiales. Sin embargo, al transcurrir de los años se ha encontrado que los colorantes artificiales pueden causar enfermedades en el ser humano, por lo que se hace necesario realizar estudios para extraer y aplicar de nuevo los tintes naturales. Se realizó la extracción y caracterización del tinte natural del Yacushapana, para luego aplicar en el proceso de tinción de telas de algodón previamente mordentadas, obteniéndose diferentes colores de acuerdo a mordiente utilizado, que son los siguientes:Marrón claro con sulfato de aluminio, amarillo pálido con sulfato de cobre, amarillo claro con sulfato de zinc. y amarillo oscuro con sulfato de fierro.
\end{abstract}

Palabras claves: yacushapana, flavonoides, xantonas, quinonas, carotenoides, mordentadas

\section{COTTON DYED WITH YUCUSHAPANA NATURAL PIGMENT USING DIFFERENT MORDANTS}

\begin{abstract}
This project is part of the research on the transformation of natural resources running in the Faculty of Chemical Engineering at the UNCP. In order to evaluate the potential for staining dye obtained from the forest species yacushapana (Terminalia oblonga) in the implementation of staining of cotton. The Peruvian jungle has a rich vegetation, including plants with active substances such as flavonoids dye, xanthones, quinones, carotenoids, etc., Substances that can be used in cosmetics, food and textiles. This project investigated the tree species with potential for use in the textile industry in the dyeing of fibers. Today the native communities of the low jungle of the Ucayali region, using aqueous dyeing extracts of plant diversity for the dyeing of the fibers used in making variety of clothing and bedding. The above aqueous dyeing extracts are obtained from an empirical way so they have a very low half-life and limited availability at certain times of year as the results of this project will be to advise entrepreneurs in the textile industry in extraction, drying, preservation and application of natural dyes. Formerly dyeing extracts of plants used for the dyeing, clothing, food coloring and so on., Which ceased to be made to appear artificial colors. However, over the years has found that artificial colors can cause disease in humans, so research is needed to remove and reapply the natural dyes. Was extracted and characterization Yacushapana natural dye, then applied in the dyeing process of cotton fabrics pre-etched, resulting in different colors according to mordant used, which are as follows: Light brown with aluminum sulfate, pale yellow copper sulfate, clear yellow zinc sulfate. and dark yellow iron sulfate.
\end{abstract}

Key words: yacushapana, flavonoids, xanthones, quinones, carotenoids, etched

\section{INTRODUCCIÓN}

36

Trabajo de investigación fue recibido el 07/05/2010 retornado para su revisión el 15/06/2010 y aprobado para su publicación 16/11/2010. 
El Perú tiene una gran fuente de biodiversidad, que el campesino en su afán de poder supervivir la toma estos recursos naturales para sacarle provecho y de alguna forma lo trata de industrializar.

El algodón es un cultivo de la zona de costa y selva, habiéndose incrementado su producción a gran escala y de él se obtiene la fibra destinada para la industria textil y algunos aceites extraídos de sus semillas, este algodón se destaca por su finura y longitud de fibra, por las condiciones especiales que da la tierra en donde lo cultivan como es el pima y el tangüis, que es muy apreciado en el mercado internacional.

El nativo de la selva para el teñido de sus prendas de vestir, toma como materia prima entre otras la corteza de los árboles, siendo el tema de investigación de este trabajo la yacushapana, que crece en la selva peruano, y sobre todo con mayor incidencia en la región de Ucayali.

La yacushapana es aplicada como pigmento para el teñido de las prendas de algodón, que los nativos de la selva usan, y este tenido no tiene buena solidez, descargando el color en las primeras lavadas de la prenda.

Debido a estas consideraciones el presente estudio tiende a realizar el trabajo experimental del teñido de prendas de algodón, con pigmentos de yacushapana, con una metodología en donde se emplean diferentes mordientes, para obtener una buena solidez en las prendas de algodón.

\section{Hipótesis de trabajo.}

El teñido de algodón con pigmento natural yucushapana es posible utilizando diferentes mordientes.

\section{Objetivos del proyecto.}

Teñido de algodón con pigmento natural de yacushapana, aplicando diferentes mordientes

\section{MATERIALES Y MÉTODOS}

\section{Reactivos y materiales \\ Materiales}

- Sistema extractor Soxhlet : 1

- Secador eléctrico de bandejas

- Refrigerante: 1

- Balón fondo redondo $1000250 \mathrm{ml}: 1$

- Mangueras: 2

- Mechero o manto calefactor.

- Trípode: 1

- Rejilla de porcelana: 1
- Pinzas: 3

- Dobles tornillos: 3

- Papel filtro.

- Vidrio reloj: 1

- Balanza analítica.

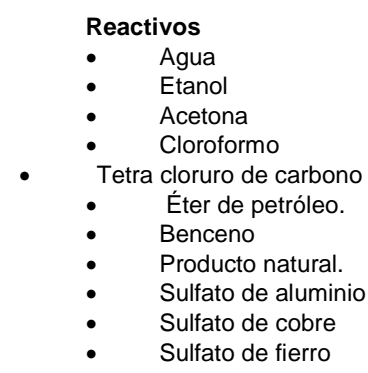

\section{Metodología}

Localización:

La parte experimental de la investigación se llevó a cabo en la Universidad Nacional del Centro del Perú, en las siguientes instalaciones:

1. Laboratorio de tecnología de la Facultad de Ingeniería Química donde se encuentra instalado el Extractor soxhlet

2. Secador solar en la facultad.

3. Secador eléctrico instalado en la Facultad de Ingeniería Química, laboratorio de tecnología.

\section{Obtención de las muestras:}

La yacushapana se obtuvo a través de la organización de nativos del Departamento de Ucayali. Se colectó corteza y se colocaron en recipientes herméticos para luego ser llevadas hacia el secador eléctrico de flujo transversal con bandejas, controlándose la humedad relativa y temperatura en el proceso de secado.

Las cortezas secas se molieron en un molino de martillos y se sometieron al proceso de extracción de colorantes naturales, destilación con reflujo a nivel laboratorio y maceración.

\section{Diseño de tratamientos}

Para la evaluación estadística se utilizó un diseño completamente al azar con un arreglo combinatorio, en el cual se aplicaron un experimento factorial evaluando 2 muestras y 3 tipos de solventes (agua, etanol al 35\% y etanol al $70 \%$ ), con 7 repeticiones para cada una, resultando 6 unidades experimentales, y un total de 42 tratamientos. El tamaño de lote fue fijo con una relación materia seca/solvente de 1:10, tiempo de extracción de 1 hora y a temperatura de ebullición. Obtenidos los extractos éstos se aplicaron en fibras de algodón utilizando el método de tinción descrito. 


\section{Manejo del experimento:}

La materia prima de yacushapana se recolectó en los bosques del departamento de Ucayali, luego se procedieron a secar en el secador eléctrico de bandejas, se colocaron en recipientes herméticos, para luego ser reducidas de tamaño en un molino de martillos. Para las extracciones a nivel laboratorio se utilizó el extractor soxhelt de $500 \mathrm{ml}$., los extractos obtenidos se secaron en el secador eléctrico de bandejas y luego se procedieron a moler en molino de martillos y se obtuvo los extractos en forma de polvo de color ámbar brillante, los que se colocaron en recipientes cerrados debidamente etiquetados y se guardaron hasta utilizarlos en el proceso de tinción.

\section{Metodología Experimental}

Método de extracción del tinte natural

Se trabajó a nivel de laboratorio se utilizó el mismo método.

Método de extracción del tinte natural a nivel laboratorio

- Se pesa un vidrio reloj con un papel filtro y, sobre éste, se pesan $15 \mathrm{~g}$. el producto natural desde el que se va extraer el pigmento (la corteza) seco y finamente dividido.

- Se envuelve firmemente el sólido en el papel filtro y se pone en el tubo Soxhlet.

- En el balón, previamente pesado, se ponen $150 \mathrm{ml}$. del solvente, medidos con probeta.

- Se conecta el refrigerante y se hace circular agua en el sentido ascendente.

- Antes de empezar a calentar a baño maría, se verifica si hay alguna fuga de solvente 0 de la corteza..

- Se mantiene el calentamiento durante al balón hasta que la cámara que contiene a la corteza se decolore y no arroje ningún color el solvente.

- Después de extraer el colorante, se elimina el solvente y concentrando el pigmento extraído.

- El pigmento extraído se pesa después de cada tratamiento.

- Se deja enfriar y, cuando el sistema está a temperatura ambiente, se saca el papel con el sólido, se abre y se coloca bajo campana, sobre el vidrio reloj, para que se seque al aire.

- Se arma nuevamente el sistema, que ahora se utilizará para separar el otro solvente del soluto extraído: se vuelve a calentar $y$, antes que el disolvente alcance el nivel del sifón, se corta el calentamiento. Se espera que esté a temperatura ambiente, se abre y se elimina el solvente.

- Se procedió a filtrar.

Los extractos obtenidos se colocaron en recipientes cerrados color ámbar para su posterior caracterización y utilización en el proceso de tinción de fibras.

- El tiempo de extracción de solvente fue continuo, hasta que la muestra no tuvo presencia de solvente. El residuo obtenido contiene extracto de colorante, el que fue almacenado en viales debidamente identificados y en refrigeración.

Después de obtener los extractos se le realizaron pruebas físicas y químicas, por medio de técnicas de identificación de flavonoides,

Métodos para la caracterización de los tintes naturales determinación de propiedades fisicoquímicas de los extractos colorantes

\section{Determinación de densidad:}

La determinación de la densidad de los extractos tintóreos se realizó con un picnómetro de $10 \mathrm{ml}$ a temperatura de 20 ${ }^{\circ} \mathrm{C}$. del extracto de agua y etanol ( $35 \%$ y $70 \%$ ).

\section{Determinación del índice de refracción:}

Se utilizó un refractómetro Abbe. Se limpió con Xilol y se vertió una gota del extracto tintóreo en el prisma, tomando nota de la lectura del aparato.

\section{Identificación de flavonoides:}

\section{Reacciones coloridas:}

Para identificar flavonoides se utilizaron las siguientes pruebas:

a. Reacción de Shinoda: al extracto alcohólico incoloro o ligeramente amarillo se le colocó un pequeño trozo de magnesio y unas pocas gotas de $\mathrm{HCl}$ concentrado el desarrollo inmediato de coloración es indicativo de la presencia de flavonas y flavonoles (amarillo a rojo), flavanonoles (rojo a magenta) flavanonas (rojo, magenta, violeta, azul), isoflavonas (amarillo), isoflavononas, chalconas y auronas no dan coloración.

b. Reacción con $\mathrm{H} 2 \mathrm{SO} 4$ concentrado a la muestra se le agregó una gota de ácido y se observa si hay cambio de color: las flavonas y flavonoles dan coloraciones fuertemente amarillas, las flavanonas, anaranjadas 0 guindas; las chalconas y auronas, rojo guinda 0 rojo azulado.

\section{Método para tinción del algodón.}

a) Se lavó el algodón con jabón neutro y agua a $30^{\circ} \mathrm{C}$, incrementar la temperatura del agua.

b) Preparar el mordiente (si es necesario) de la siguiente manera:

Por cada kilo de algodón:

b.1 Pesar $100 \mathrm{~g}$ de sulfato de aluminio

b.2 Pesar $60 \mathrm{~g}$ de sulfato de cobre

b. 3 Pesar $60 \mathrm{~g}$ de sulfato de fierro.

b. 4 Pesar $70 \mathrm{gr}$ de sulfato de zinc.

b. 5 Mezclar los reactivos con agua a $40^{\circ} \mathrm{C}$ suficiente para disolver las sales. 
c. Verter el algodón en el recipiente donde está el mordiente, aumentar paulatinamente la temperatura hasta $90^{\circ} \mathrm{C}$ por una hora, luego sacar el algodón.

d. Previamente se tiene preparado la solución tintórea a $40^{\circ} \mathrm{C}$, introducir el algodón en el recipiente y aumentar la temperatura hasta $90^{\circ} \mathrm{C}$ por una hora.

e. Esperar que se enfríe la solución.

f. Sacar el algodón y lavar con jabón neutro hasta que la materia no destiña.

g. Secar

\section{Extracción con solventes}

\section{RESULTADOS}

Tabla 4. -Rendimiento de colorante extraído

\begin{tabular}{|c|c|c|c|c|c|}
\hline SOLVENTE & FORIIULA & $\begin{array}{l}\text { PESO MUESTRA } \\
\text { Gr. }\end{array}$ & $\begin{array}{l}\text { COLOR DE COLORANTE } \\
\text { EXTRAIDO }\end{array}$ & $\begin{array}{c}\text { PESO } \\
\text { COLORANTE } \\
\text { EXTRAIDO gr. }\end{array}$ & $\begin{array}{c}\% \\
\text { RENDIENTO } \\
\text { DE } \\
\text { COLORANTE }\end{array}$ \\
\hline Agua & $\mathrm{OHH}$ & 15.0 & Marrón oscuro & 0.75 & 5 \\
\hline Etanol & $\mathrm{CH}_{3}-\mathrm{CH}_{2}-\mathrm{OH}$ & 15.0 & Marrón claro & 0.45 & 3 \\
\hline Acetona & $\stackrel{0}{\mathrm{C}}_{\mathrm{CH}_{3}-\mathrm{C}_{-} \mathrm{CH}_{3}}$ & 15.0 & Verde oscuro & 0.30 & 2 \\
\hline Cloroformo & $\mathrm{CHCl}_{3}$ & 15.0 & Verde amarillento & 0.225 & 1.5 \\
\hline $\begin{array}{l}\text { Tetracloruro } \\
\text { de carbono }\end{array}$ & $\mathrm{CCl}_{4}$ & 15.0 & $\begin{array}{l}\text { Amarrillo } \\
\text { oscuro }\end{array}$ & 0.18 & 1.2 \\
\hline Eter & $\begin{array}{c}\mathrm{CH}_{3}-\mathrm{CH}_{2}-\mathrm{O}-\mathrm{CH}_{2} \\
\mathrm{CH}_{3}\end{array}$ & 15.0 & Amarrillo claro & 0.15 & 1.0 \\
\hline Benceno & & 15.0 & Blanco & 0.12 & 0.8 \\
\hline
\end{tabular}

Análisis Estadístico Con Minitab

Tabla 5.- Análisis estadístico utilizando el paquete MINITAB en rendimiento de extracción del colorante natural

\begin{tabular}{cccc}
\hline Agua & Etanol & Tetracloruro C & Cloroformo \\
\hline 5.0 & 3.0 & 2.9 & 2.0 \\
4.5 & 3.4 & 2.0 & 1.5 \\
6.0 & 4.5 & 3.4 & 2.5 \\
4.9 & 4.9 & 2.8 & 2.8 \\
& 5.5 & 3.1 & 2.4 \\
& & 4.6 & 1.8 \\
& & 2.5 & \\
\hline
\end{tabular}

\section{RESULTADO}

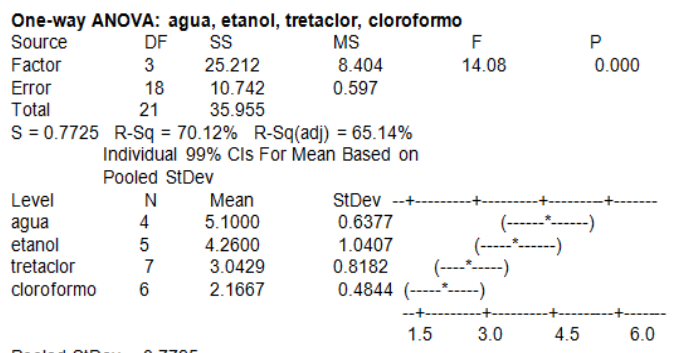

Pooled StDev $=0.7725$

El valor de $\mathrm{F}=5,18$ (Tablas) y el valor obtenido de $\mathrm{F}=$ 14,08 , siendo mayor, por lo que se confirma que el valor obtenido de cada extracto es diferente.

\section{Propiedad de densidad del extracto}

Tabla 6.- Densidad de extracto de colorante de agua y alcohol por su alto rendimiento

\begin{tabular}{|c|c|c|c|c|}
\hline Corteza & Solvente & Corrida & $\begin{array}{l}\text { Densidad } \\
\mathrm{g} / \mathrm{ml}\end{array}$ & Promedio \\
\hline & Agua & 1 & 1.0800 & \\
\hline & Etanol $35 \%$ & 2 & 1.0835 & 1.0870 \\
\hline & Etanol $70 \%$ & 3 & 1.0975 & \\
\hline & Agua & 1 & 1.0875 & \\
\hline Yacushapa & Etanol $35 \%$ & 2 & 1.1000 & 1.0980 \\
\hline \multirow[t]{4}{*}{ na } & Etanol $70 \%$ & 3 & 1.1050 & \\
\hline & Agua & 1 & 1.0815 & \\
\hline & Etanol $35 \%$ & 2 & 1.0990 & 1.0880 \\
\hline & Etanol $70 \%$ & 3 & 1.0825 & \\
\hline
\end{tabular}

Reacción de coloración con ácido sulfúrico de flavonoides.

Tabla 7.- Reacción colorida con ácido sulfúrico para la identificación de flavonoides en los extractos colorantes.

\begin{tabular}{lcc}
\hline ESPECIE & SOLVENTE & $\begin{array}{l}\text { FLAVONOIDE } \\
\text { IDENTIFICACION }\end{array}$ \\
\hline \multirow{3}{*}{ Yacushapan } & Agua & Flavonas y flovonoles \\
& Etanol $35 \%$ & Flavonas y flovonoles \\
& Etanol $70 \%$ & Flavonas y flovonoles \\
\hline
\end{tabular}

Teñido De Yacushapana Con Diferentes Mordientes

Tabla 8.- Teñido del algodón con diferentes mordientes

\begin{tabular}{|c|c|c|c|c|c|c|}
\hline $\begin{array}{l}\text { Peso de } \\
\text { tela (gr) }\end{array}$ & Mordiente & $\begin{array}{l}\text { Peso de } \\
\text { mordiente } \\
\text { (gr/ml) }\end{array}$ & $\begin{array}{c}\text { Tiempo de } \\
\text { mordentad } \\
\text { o (min) }\end{array}$ & $\begin{array}{c}\text { Tiempo de } \\
\text { tinción } \\
\text { (min) }\end{array}$ & $\begin{array}{l}\text { Color } \\
\text { obtenido }\end{array}$ & $\begin{array}{c}\text { Resultad } \\
0 \\
\text { obtenido } \\
\end{array}$ \\
\hline 2 & $\mathrm{Al}_{2}\left(\mathrm{SO}_{4}\right)_{3}$ & $100 / 1000$ & 30 & 60 & Uarrón claro & Eficiente \\
\hline 2 & $\mathrm{Cu}_{2} \mathrm{SO}_{4}$ & $60 / 1000$ & 25 & 60 & $\begin{array}{c}\text { Amarillo } \\
\text { pálido }\end{array}$ & Bueno \\
\hline 2 & $\mathrm{ZnSO}_{4}$ & $60 / 1000$ & 25 & 60 & $\begin{array}{r}\text { Amarillo } \\
\text { claro }\end{array}$ & Regular \\
\hline 2 & $\mathrm{Fe}_{2}\left(\mathrm{SO}_{4}\right)_{3}$ & $70 / 1000$ & 30 & 60 & $\begin{array}{r}\text { Amarrillo } \\
\text { oscuro }\end{array}$ & Bueno \\
\hline
\end{tabular}

\section{DISCUSIÓN}

\section{Extracción con solventes}

- Se empleó los solventes tomando como referencia su polaridad siendo el más polar el agua y el menos polar el benceno.

- De acuerdo a esta polaridad el colorante extraído nos dio una coloración característica del extracto.

- En función del solvente se logró extraer diferentes pesos de colorante, no obstante de haber empleado las mismas cantidades de peso de corteza, el volumen del solvente.

- Después de haber hecho la extracción se calculó el rendimiento que arrojó de colorante, en función del solvente aplicado, en donde se obtuvo como resultado de $5 \%$ de rendimiento de colorante con el solvente agua.

- Los resultados obtenidos se muestran en la tabla 4. 


\section{Análisis Estadístico con Minitab}

De lo que se concluye que el valor calculado de F 14.08, que es mayor que el valor crítico 5.09 de tablas, por lo tanto se rechaza la hipótesis nula. Se concluye que las medias poblacionales de rendimiento no son iguales.

Es probable que los rendimientos que se obtuvieron en la extracción estén relacionados con la polaridad del solvente. Por lo que se concluye que hay diferencias entre las medias de tratamiento, como se muestra en la tabla 5

\section{Propiedad de densidad del extracto}

Para los valores de densidad obtenidos, solo hay efecto significativo del solvente, por lo que el agua es menos densa, con respecto a la densidad del extracto con alcohol ( $35 \%$ y $70 \%$ ), como se muestra en la tabla 6

\section{Reacción de coloración con ácido sulfúrico de flavonoides}

Los colorantes naturales que por sus características físicas se clasifican como pigmentos, comprenden numerosos tipos de sustancias, que por lo general se dividen en dos grandes grupos. El primer grupo abarca aquellos pigmentos que contienen Nitrógeno, como las Hemoglobinas, las clorofilas. El segundo grupo está formado por pigmentos sin Nitrógeno. Los Carotenoides son miembros de este grupo, como lo son los pigmentos vegetales llamados Flavonoides. Se observa en el extracto colorante de la corteza de yacushapana están presentes los Flavonoides denominados Flavonas y Flavonoles, independiente del tipo de solvente utilizado. Como se describe en el cuadro 7

\section{Teñido de Yacushapana con diferentes mordientes}

En la fibra de algodón teñida con colorante de yacushapana, se observó solo un ligero oscurecimiento de color marrón claro cuando se aplica sulfato de aluminio y siendo el color eficiente.

Cuando se aplica sulfato de cobre como mordiente a la fibra de algodón se obtiene una amarrillo pálido, con resultado bueno.

Con la aplicación de sulfato de zinc, el resultado del color teñido es regular, con resultado de color de amarrillo claro.

Aplicando sulfato de fierro, se tiene un resultado bueno en la tinción, dando una coloración de la tela de algodón de amarillo oscuro

Los resultados se muestran en la tabla 8

\section{CONCLUSIONES}

$\checkmark \quad$ El rendimiento en el extracto colorante obtenido es claramente muy diferente dependiendo del solvente que se trate, ya que se obtuvo un valor promedio de $5.0 \%$ en agua que es la mayor y $0.8 \%$ de benceno que es la menor, respectivamente.

$\checkmark \quad$ En el rendimiento del extracto colorante obtenido de la especies hay influencia del solvente utilizado y de la interacción entre ellos. En cuanto al solvente, el etanol claramente produce menor rendimiento que el agua.

$\checkmark$ Hay efectos significativos de especie, solvente y su interacción.

$\checkmark$ Solo hay efecto significativo del solvente, aunque también hay que juzgar si esta diferencia tiene sentido práctico.

$\checkmark \quad$ La densidad del extracto en alcohol (30 \% y $70 \%$ ) es mayor que lo extraído con el agua.

$\checkmark \quad$ Para el reconocimiento del extracto se aplicó ácido sulfúrico, lo cual nos indica la presencia de Flavonas y flovonoles.

$\checkmark \quad$ Como resultado de la aplicación de mordiente a la tela de algodón blanqueado se obtuvo las siguientes coloraciones:

- Marrón claro con sulfato de aluminio

- Amarillo pálido con sulfato de cobre

- Amarillo claro con sulfato de zinc. Y

- Amarillo oscuro con sulfato de fierro.

\section{REFERENCIAS BIBLIOGRÁFICAS}

- Domínguez, X. A; Métodos de investigación Fotoquímica, México, editorial Limusa Wiley, 1983.

- Lock de Ugaz, Olga; Investigaciones Fotoquímicas, métodos en el estudio de Productos naturales, Perú, Editorial de la pontificia Universidad Católica del Perú, segunda edición, 1994-

- Gibaja, S; Guía para el análisis de los compuestos del carbono, Perú, Editorial de la Universidad Mayor de san Marcos, 1997-

- Allinger L. Norman; Cavs P. Michel; Química Orgánica, México Editorial Reverte Segunda edición, 2001.

- Asociación peruana de Facultades y escuelas de Química e Ingeniería Química; técnicas de Caracterización de Compuestos, Perú, Editorial Universidad Mayor de san Marcos, 1995. 